\title{
Boundedness and Oscillation of Third Order Neutral Differential Equations with Deviating Arguments
}

\author{
Elmetwally M. Elabbasy, Magdy Y. Barsoum, Osama Moaaz \\ Department of Mathematics, Faculty of Science, Mansoura University, Mansoura, Egypt \\ Email: emelabbasy@mans.edu.eg,o_moaaz@mans.edu.eg
}

Received 15 June 2015; accepted 16 November 2015; published 19 November 2015

Copyright @ 2015 by authors and Scientific Research Publishing Inc.

This work is licensed under the Creative Commons Attribution International License (CC BY).

http://creativecommons.org/licenses/by/4.0/

(c) $\underset{\mathrm{EY}}{\mathrm{B}}$ Open Access

\section{Abstract \\ we consider the third-order neutral functional differential equations with deviating argu- ments. A new theorem is presented that improves a number of results reported in the literature. Examples are included to illustrate new results.}

Keywords

Oscillation, Third Order, Neutral Delay Differential Equations

\section{Introduction}

In this paper we consider third order neutral differential equations of the form

$$
\left(r_{2}(t)\left(r_{1}(t) z^{\prime}(t)\right)^{\prime}\right)^{\prime}+\int_{\alpha}^{\beta} q(t, \xi) f(x(g(t, \xi))) \mathrm{d} \sigma(\xi)=0, t \geq t_{0},
$$

where $z(t)=x(t) \pm p(t) x(\tau(t))$ and the following conditions are satisfied

$\left(\mathrm{A}_{1}\right) \quad p \in C(I, \mathbb{R}), 0<p(t) \leq p<1$ and $I=\left[t_{0}, \infty\right)$,

$\left(\mathrm{A}_{2}\right) \tau \in C(I, \mathbb{R}), \tau(t)<t, \tau$ is strictly increasing, $\lim _{t \rightarrow \infty} \tau(t)=\infty$ and we define

$\tau^{0}(t)=t, \tau^{j}(t)=\tau\left(\tau^{j-1}(t)\right), j=1,2, \cdots$,

$\left(\mathrm{A}_{3}\right) r_{i} \in C(I,(0, \infty)), \int_{t_{0}}^{\infty} r_{i}^{-1}(t) \mathrm{d} t=\infty, i=1,2$,

$\left(\mathrm{A}_{4}\right) \quad f \in C(\mathbb{R}, \mathbb{R}), f$ is non-decreasing and $u f(u)>0$ for $u \neq 0$, 
$\left(\mathrm{A}_{5}\right) q \in C(I \times[\alpha, \beta],[0, \infty)]$, and $q(t, \xi)$ is not zero on any half line $\left[t_{\mu}, \infty\right) \times[\alpha, \beta], t_{\mu} \geq t_{0}$,

$\left(\mathrm{A}_{6}\right) \quad g \in C(I \times[\alpha, \beta], \mathbb{R}), g(t, \xi) \leq t$ for $t \geq t_{0}$ and $\xi \in[\alpha, \beta], g(t, \xi)$ is continuous, has positive partial derivative on $I \times[\alpha, \beta]$ with respect to $t$, nondecreasing with respect to $\xi$ and $\lim g(t, \xi)=\infty$,

$\left(\mathrm{A}_{7}\right) \sigma \in C([\alpha, \beta], \mathbb{R}), \sigma$ is nondecreasing and the integral of Equation (1) is in the senne Riemann-stieltijes.

We mean by a solution of Equation (1) a function $x(t):\left[t_{x}, \infty\right) \rightarrow \mathbb{R}, t_{x} \geq t_{0}$ such that $z(t), r_{1}(t) z^{\prime}(t)$, $r_{2}(t)\left(r_{1}(t) z^{\prime}(t)\right)^{\prime}$ and $\left(r_{2}(t)\left(r_{1}(t) z^{\prime}(t)\right)^{\prime}\right)^{\prime}$ exist and are continuous on $\left[t_{x}, \infty\right)$. A nontrivial solution of (1) is called oscillatory if it has arbitrarily large zeros, otherwise it is called non-oscillatory.

Asymptotic properties of solutions of differential equations of the second and third order have been subject of intensive studying in the literature. This problem for neutral differential equations has received considerable attention in recent years (see [1]-[11]).

Recently, in [12] by using Riccati technique, have established some general oscillation criteria for third-order neutral differential equation

$$
\left(r_{2}(t)\left(r_{1}(t)(x(t)+p x(t-\tau))^{\prime}\right)^{\prime}\right)^{\prime}+q(t) x(t-\sigma)=0 .
$$

In [3], Candan presented several oscillation criteria for third order neutral delay differential equation

$$
\left(r_{2}(t)\left(r_{1}(t)(x(t)+p x(t-\tau))^{\prime}\right)^{\prime}\right)^{\prime}+\int_{\alpha}^{\beta} q(t, \xi) x(g(t, \xi)) \mathrm{d} \sigma(\xi)=0 .
$$

[9] and [13] obtained some oscillation criteria for study third order nonlinear neutral differential equations

$$
\left(r_{2}(t)\left(r_{1}(t)(x(t)+p x(t-\tau))^{\prime}\right)^{\prime}\right)^{\prime}+q(t) f(x(t-\sigma))=0
$$

and

$$
\left(r_{2}(t)\left(r_{1}(t)(x(t)-p x(\tau(t)))^{\prime}\right)^{\prime}\right)^{\prime}+q(t) f(x(g(t)))=0 .
$$

In this paper, we establish some oscillation criteria for Equation (1), which complement and extend the results in [3] [13].

We begin with analyzing of the asymptotic behavior of possible non-oscillatory solutions of the Equation (1) in the case when $z(t)=x(t)-p x(\tau(t))$. Let $x(t)$ be a non-oscillatory solution of (1) on $\left[t_{0}, \infty\right)$. From (1) it follows that the function $z(t)$ has to be eventually of constant sign, so either

(a) $x(t) z(t)>0$

or

(b) $x(t) z(t)<0$

for all sufficiently large $t$. Denote by $N^{+}$[or $N^{-}$] the set of all non-oscillatory solutions $x(t)$ of the Equation (1) such that (a) [or (b)] is satisfied. We begin with some useful lemmas.

Lemma 1.1 Let $z(t)=x(t)-p x(\tau(t))$. Assume that $\left(\mathrm{A}_{1}\right)$ and $\left(\mathrm{A}_{2}\right)$ hold and $x$ be continuous non-oscillatory solution of the functional inequality (a). Then

$$
\lim _{t \rightarrow \infty} x(t)=0 \text { and } \lim _{t \rightarrow \infty} z(t)=0 .
$$

Lemma 1.2 Let $z(t)=x(t)-p x(\tau(t))$. Assume that $\left(\mathrm{A}_{1}\right)$ and $\left(\mathrm{A}_{2}\right)$ hold and $x$ be continuous non-oscillatory solution of the functional inequality (b). If $\lim _{t \rightarrow \infty} z(t)=0$, then

$$
\lim _{t \rightarrow \infty} x(t)=0
$$


These lemmas are modifications of the Lemma 1 in the paper [14] and the Lemma 2 in the paper [13].

\section{Main Results}

In this part, for the sake of convenience, we introduce the following notation:

$$
\Theta(t)=\int_{\alpha}^{\beta} q(t, \xi) \mathrm{d} \sigma(\xi) \text { and } R(t)=\int_{t_{0}}^{t} r_{2}^{-1}(s) \mathrm{d} s .
$$

\subsection{Oscillation Criteria If $\mathrm{Z}(\boldsymbol{t})=x(t)-p x(\tau(t))$}

In this section, we will establish some oscillation criteria for Equation (1) in the case when $z(t)=x(t)-p x(\tau(t))$ and $p(t)=p$.

Lemma 2.1 Let $x$ be a bounded positive solution of Equation (1) on the interval I. Then there exists $a T_{0} \geq t_{0}$ such that $z(t)$ has the following properties:

$$
z^{\prime}(t)<0,\left(r_{1}(t) z^{\prime}(t)\right)^{\prime}>0 \text { and }\left(r_{2}(t)\left(r_{1}(t) z^{\prime}(t)\right)^{\prime}\right)^{\prime} \leq 0 \text { for } t \geq T_{0}
$$

Proof. Let $x$ be a bounded positive solution of Equation (1) on the interval $I$. From $\left(\mathrm{A}_{1}\right),\left(\mathrm{A}_{2}\right)$ and $\left(\mathrm{A}_{6}\right)$, there exists a $t_{1} \geq t_{0}$ such that $x(t)>0, x(\tau(t))>0$ and $x(g(t, \xi))>0$ for $t \geq t_{1}$. Then $z(t)$ is bounded and non-oscillatory. Thus, Equation (1) implies that

$$
\left(r_{2}(t)\left(r_{1}(t) z^{\prime}(t)\right)^{\prime}\right)^{\prime}=-\int_{\alpha}^{\beta} q(t, \xi) f(x(g(t, \xi))) \mathrm{d} \sigma(\xi) \leq 0
$$

Hence, the function $r_{2}(t)\left(r_{1}(t) z^{\prime}(t)\right)^{\prime}$ is a non-increasing and of one sign. We claim that $r_{2}(t)\left(r_{1}(t) z^{\prime}(t)\right)^{\prime}>0$ for $t \geq t_{1}$. Suppose that $r_{2}(t)\left(r_{1}(t) z^{\prime}(t)\right)^{\prime}<0$ for $t \geq t_{2} \geq t_{1}$. Then there exists a $t_{3} \geq t_{2}$ and constant $K_{1}>0$ such that

$$
r_{2}(t)\left(r_{1}(t) z^{\prime}(t)\right)^{\prime}<-K_{1} \text { for } t \geq t_{3} .
$$

By integrating the last inequality from $t_{3}$ to $t$, we get

$$
r_{1}(t) z^{\prime}(t)<r_{1}\left(t_{3}\right) z^{\prime}\left(t_{3}\right)-K_{1} \int_{t_{3}}^{t} \frac{1}{r_{2}(s)} \mathrm{d} s .
$$

Letting $t \rightarrow \infty$, from $\left(\mathrm{A}_{3}\right)$, we have $\lim _{t \rightarrow \infty} r_{1}(t) z^{\prime}(t)=-\infty$. Then there exists a $t_{4} \geq t_{3}$ and constant $K_{2}>0$ such that

$$
r_{1}(t) z^{\prime}(t)<-K_{2} \text { for } t \geq t_{4}
$$

By integrating this inequality from $t_{4}$ to $t$ and using $\left(\mathrm{A}_{3}\right)$, we get $\lim _{t \rightarrow \infty} z(t)=-\infty$. This yields that $x \in N^{-}$ and this contradicts the Lemma 1.1. Now we have $r_{2}(t)\left(r_{1}(t) z^{\prime}(t)\right)^{\prime}>0$ for $t \geq t_{2}$. Hence $r_{1}(t) z^{\prime}(t)$ is increasing function and we have two possible cases for $r_{1}(t) z^{\prime}(t)$ either $r_{1}(t) z^{\prime}(t)>0$ eventually or $r_{1}(t) z^{\prime}(t)<0$ eventually for $t \geq t_{3} \geq t_{2}$. If $r_{1}(t) z^{\prime}(t)>0$ for $t \geq t_{3}$, then there exist a $t_{4} \geq t_{3}$ and a constant $L>0$ such that

$$
r_{1}(t) z^{\prime}(t)>L \text { for } t \geq t_{4}
$$

By integrating this inequality from $t_{4}$ to $t$ and using $\left(\mathrm{A}_{3}\right)$, we get $\lim _{t \rightarrow \infty} z(t)=\infty$. This means that $z(t)>0$ and we get $x(t) \geq z(t)$ for all sufficiently large $t$. Then $\lim _{t \rightarrow \infty} z(t)=\infty$, which contradicts the boundedness of $x(t)$. Hence, $z^{\prime}(t)<0$ for $t \geq t_{3}$. 


\section{Theorem 2.1 if}

$$
\limsup _{t \rightarrow \infty} \int_{g(t, \beta)}^{t} \Theta(s) \int_{g(s, \beta)}^{g(t, \beta)} \frac{1}{r_{1}(u)} \int_{u}^{g(t, \beta)} \frac{1}{r_{2}(v)} \mathrm{d} v \mathrm{~d} u \mathrm{~d} s>0 .
$$

Then every bounded solution $x(t)$ of Equation (1) is either oscillatory or tends to zero.

Proof. Let $x$ be a bounded non-oscillatory solution of Equation (1) on the interval $I$. Without loss of generality we may assume that $x(t)>0$. From Lemma 2.1, we get that (2) holds. New, we have

$$
x(t)=z(t)+p x(\tau(t))=z(t)+p z(\tau(t))+p^{2} x\left(\tau^{2}(t)\right),
$$

for all sufficiently large $t$. Repeating this procedure and the monotonicity of $z$, we obtain that there exists an integer $n \geq 0$ such that $\tau^{n+1}(g(t, \xi)) \geq T_{0}$ and

$$
x(g(t, \xi))=\sum_{r=0}^{n} p^{r} z\left(\tau^{r}(g(t, \xi))\right)+p^{n+1} x\left(\tau^{n+1}(g(t, \xi))\right) \geq \lambda z(g(t, \xi)),
$$

where $\lambda=\sum_{r=0}^{n} p^{r}=\frac{1-p^{n+1}}{1-p}>1$. Hence, we get

$$
f(x(g(t, \xi))) \geq f(\lambda z(g(t, \xi))) \geq f(z(g(t, \xi))) \geq f(z(g(t, \beta))) .
$$

Thus, from Equation (1), we obtain

$$
\left(r_{2}(t)\left(r_{1}(t) z^{\prime}(t)\right)^{\prime}\right)^{\prime} \leq-f(z(g(t, \beta))) \Theta(t) .
$$

Now, since $z(t)$ is bounded decreasing function, then there exist $\gamma$ such that

$$
\lim _{t \rightarrow \infty} z(t)=\gamma \text {. }
$$

If $z(t)<0$ for $t \geq T_{1} \geq T_{0}$, then $\gamma<0$ and which contradicts the Lemma 1.1. Therefore $z(t)>0$ for $t \geq T_{1}$ and $\gamma \geq 0$. We shall prove that $\gamma=0$. Let $\gamma>0$. For $t>u>T \geq T_{1}$, we obtain

$$
\int_{u}^{g(t, \beta)} \frac{1}{r_{2}(v)}\left[r_{2}(v)\left(r_{1}(v) z^{\prime}(v)\right)^{\prime}\right] \mathrm{d} v=r_{1}(g(t, \beta)) z^{\prime}(g(t, \beta))-r_{1}(u) z^{\prime}(u) .
$$

Thus, form Lemma 2.1, we get

$$
\begin{aligned}
r_{1}(u) z^{\prime}(u) & \leq-\int_{u}^{g(t, \beta)} \frac{1}{r_{2}(v)}\left[r_{2}(v)\left(r_{1}(v) z^{\prime}(v)\right)^{\prime}\right] \mathrm{d} v \\
& \leq-\left[r_{2}(g(t, \beta))\left(r_{1}(g(t, \beta)) z^{\prime}(g(t, \beta))\right)^{\prime}\right] \int_{u}^{g(t, \beta)} \frac{1}{r_{2}(v)} \mathrm{d} v .
\end{aligned}
$$

So, for $t>u>s>T$, we have

$$
z(g(t, \beta))-z(g(s, \beta))=\int_{g(s, \beta)}^{g(t, \beta)} \frac{1}{r_{1}(u)}\left[r_{1}(u) z^{\prime}(u)\right] \mathrm{d} u .
$$

Hence, from (6), we get

$$
z(g(t, \beta))-z(g(s, \beta)) \leq-G(g(t, \beta)) \int_{g(s, \beta)}^{g(t, \beta)} \frac{1}{r_{1}(u)} \int_{u}^{g(t, \beta)} \frac{1}{r_{2}(v)} \mathrm{d} v \mathrm{~d} u
$$

where $G(y)=r_{2}(y)\left(r_{1}(y) z^{\prime}(y)\right)^{\prime}$. Let us define function

$$
F(s, t)=(G(s)-G(g(t, \beta))) \int_{s}^{t} \frac{z^{\prime}(g(u, \beta)) g^{\prime}(u, \beta)}{f(z(g(u, \beta)))} \mathrm{d} u, t \geq s \geq T
$$


We note that $F(t, t)=0=F(g(t, \beta), t)$. Deriving $F(s, t)$ partially with respect to $s$ and using Lemma 2.1, $\left(\mathrm{A}_{4}\right)$ and $\left(\mathrm{A}_{6}\right)$, we get

$$
\begin{aligned}
F_{s}^{\prime}(s, t) \geq & G^{\prime}(s) \int_{s}^{t} \frac{z^{\prime}(g(u, \beta)) g^{\prime}(u, \beta)}{f(z(g(u, \beta)))} \mathrm{d} u \\
& +G(g(t, \beta)) \frac{z^{\prime}(g(s, \beta)) g^{\prime}(s, \beta)}{f(z(g(s, \beta)))}
\end{aligned}
$$

From (5), we have $G^{\prime}(s) \leq-f(z(g(s, \beta))) \Theta(s)$. Hence, we obtain

$$
\begin{aligned}
F_{s}^{\prime}(s, t) \geq & -\Theta(s) f(z(g(s, \beta))) \int_{s}^{t} \frac{z^{\prime}(g(u, \beta)) g^{\prime}(u, \beta)}{f(z(g(u, \beta)))} \mathrm{d} u \\
& +G(g(t, \beta)) \frac{z^{\prime}(g(s, \beta)) g^{\prime}(s, \beta)}{f(z(g(s, \beta)))}
\end{aligned}
$$

By $\left(\mathrm{A}_{4}\right)$ and $\left(\mathrm{A}_{6}\right)$, we get

$$
\begin{aligned}
\int_{s}^{t} \frac{z^{\prime}(g(u, \beta)) g^{\prime}(u, \beta)}{f(z(g(u, \beta)))} \mathrm{d} u & \leq \frac{1}{f(z(g(s, \beta)))} \int_{s}^{t} \mathrm{~d} z(g(u, \beta)) \\
& =\frac{1}{f(z(g(s, \beta)))}(z(g(t, \beta))-z(g(s, \beta))) \leq 0
\end{aligned}
$$

Thus, from (7), we have

$$
\int_{s}^{t} \frac{z^{\prime}(g(u, \beta)) g^{\prime}(u, \beta)}{f(z(g(u, \beta)))} \mathrm{d} u \leq \frac{-G(g(t, \beta))}{f(z(g(s, \beta)))} \int_{g(s, \beta)}^{g(t, \beta)} \frac{1}{r_{1}(u)} \int_{u}^{g(t, \beta)} \frac{1}{r_{2}(v)} \mathrm{d} v \mathrm{~d} u .
$$

Then, substituting (8) in (9), it follows that

$$
F_{s}^{\prime}(s, t) \geq G(g(t, \beta))\left[\Theta(s) \int_{g(s, \beta)}^{g(t, \beta)} \frac{1}{r_{1}(u)} \int_{u}^{g(t, \beta)} \frac{1}{r_{2}(v)} \mathrm{d} v \mathrm{~d} u+\frac{z^{\prime}(g(s, \beta)) g^{\prime}(s, \beta)}{f(z(g(s, \beta)))}\right] .
$$

By integrating this inequality from $g(t, \beta)$ to $t$ with respect to $s$, we obtain

$$
0 \geq \int_{g(t, \beta)}^{t} \Theta(s) \int_{g(s, \beta)}^{g(t, \beta)} \frac{1}{r_{1}(u)} \int_{u}^{g(t, \beta)} \frac{1}{r_{2}(v)} \mathrm{d} v \mathrm{~d} u \mathrm{~d} s+\int_{z(g(g(t, \beta), \beta))}^{z(g(t, \beta))} \frac{\mathrm{d} \omega}{f(\omega)} .
$$

where $G(g(t, \beta))>0$. Since $\lim _{t \rightarrow \infty} z(t)=\gamma>0$, we get

$$
\lim _{t \rightarrow \infty} \int_{z(g(g(t, \beta), \beta))}^{z(g(t, \beta))} \frac{\mathrm{d} \omega}{f(\omega)}=0
$$

Hence, from (10), we have

$$
\limsup _{t \rightarrow \infty} \int_{g(t, \beta)}^{t} \Theta(s) \int_{g(s, \beta)}^{g(t, \beta)} \frac{1}{r_{1}(u)} \int_{u}^{g(t, \beta)} \frac{1}{r_{2}(v)} \mathrm{d} v \mathrm{~d} u \mathrm{~d} s \leq 0,
$$

which contradicts (3). Therefore, $\lim _{t \rightarrow \infty} z(t)=0$ and according to the Lemma 1.2 we have that $\lim _{t \rightarrow \infty} x(t)=0$.

In the following Theorem, we establish some sufficient conditions for boundedness and oscillation of Equation (1) under the condition

$$
\liminf _{u \rightarrow 0} \frac{f(u)}{u}>0
$$


Theorem 2.2 Let (11) holds. If there exist an integer $n \geq 0$ such that

$$
\limsup _{t \rightarrow \infty} \int_{g(t, \beta)}^{t} \Theta(s) \int_{g(s, \beta)}^{g(t, \beta)} \frac{1}{r_{1}(u)} \int_{u}^{g(t, \beta)} \frac{1}{r_{2}(v)} \mathrm{d} v \mathrm{~d} u \mathrm{~d} s>\frac{1-p}{1-p^{n+1}} \limsup _{u \rightarrow 0} \frac{u}{f(u)},
$$

then every bounded solution $x(t)$ of Equation (1) is oscillatory.

Proof. Let $x$ be a bounded non-oscillatory solution of Equation (1) on the interval $I$. Without loss of generality we may assume that $x(t)>0$. We can proceed exactly as in the proof of Theorem 2.1 and we use the fact that (12) implies (3). Hence, we get a non-oscillatory solution with the properties $x(t)>0, z(t)>0, z^{\prime}(t)<0$ and $\left(r_{1}(t) z^{\prime}(t)\right)^{\prime}>0$ for $t \geq T_{0}, \lim _{t \rightarrow \infty} z(t)=0$ and $\lim _{t \rightarrow \infty} x(t)=0$. New, from (4), there exists $T_{1} \geq T_{0}$ such that

$$
f(x(g(t, \xi))) \geq f(\lambda z(g(t, \xi))) \geq f(\lambda z(g(t, \beta))) \text { for } t \geq T_{1} .
$$

Thus, Equation (1) implies that

$$
\left(r_{2}(t)\left(r_{1}(t) z^{\prime}(t)\right)^{\prime}\right)^{\prime} \leq-f(\lambda z(g(t, \beta))) \Theta(t) .
$$

By integrating this inequality from $g(t, \beta)$ to $t$, we get

$$
G(t) \leq G(g(t, \beta))-\int_{g(t, \beta)}^{t} f(\lambda z(g(s, \beta))) \Theta(s) \mathrm{d} s .
$$

where $G(y)=r_{2}(y)\left(r_{1}(y) z^{\prime}(y)\right)^{\prime}>0$. Thus, we obtain

$$
0 \leq G(g(t, \beta))-M \lambda \int_{g(t, \beta)}^{t} z(g(s, \beta)) \Theta(s) \mathrm{d} s .
$$

where $M=\inf \left\{\frac{f(u)}{u}: 0 \leq|u| \leq|\lambda z(g(t, \beta))|\right\}$. Since $z(t)>0$, from the Inequality (7), we get

$$
-Z(g(s, \beta)) \leq-G(g(t, \beta)) \int_{g(s, \beta)}^{g(t, \beta)} \frac{1}{r_{1}(u)} \int_{u}^{g(t, \beta)} \frac{1}{r_{2}(v)} \mathrm{d} v \mathrm{~d} u .
$$

Combining (13) and (14), we have

$$
0<G(g(t, \beta))\left(1-M \lambda \int_{g(t, \beta)}^{t} \Theta(s) \int_{g(s, \beta)}^{g(t, \beta)} \frac{1}{r_{1}(u)} \int_{u}^{g(t, \beta)} \frac{1}{r_{2}(v)} \mathrm{d} v \mathrm{~d} u \mathrm{~d} s\right)
$$

Hence, we get

$$
\int_{g(t, \beta)}^{t} \Theta(s) \int_{g(s, \beta)}^{g(t, \beta)} \frac{1}{r_{1}(u)} \int_{u}^{g(t, \beta)} \frac{1}{r_{2}(v)} \mathrm{d} v \mathrm{~d} u \mathrm{~d} s<\frac{1}{M \lambda},
$$

for $t \geq s \geq T_{1}$ and this contradicts the condition (12).

Corollary 2.1 Let (11) holds. If

$$
\limsup _{t \rightarrow \infty} \int_{g(t, \beta)}^{t} \Theta(s) \int_{g(s, \beta)}^{g(t, \beta)} \frac{1}{r_{1}(u)} \int_{u}^{g(t, \beta)} \frac{1}{r_{2}(v)} \mathrm{d} v \mathrm{~d} u \mathrm{~d} s>(1-p) \limsup _{u \rightarrow 0} \frac{u}{f(u)}
$$

then every bounded solution $x(t)$ of Equation (1) is oscillatory.

Example 2.1 Consider the differential equation

$$
\left(\sqrt{t}\left(\sqrt{t}\left(x(t)-\frac{1}{2} x\left((\sqrt{t}-1)^{2}\right)\right)^{\prime}\right)^{\prime}\right)^{\prime}+\int_{0}^{1} \frac{3 \xi^{2}}{\sqrt{t}} x\left((\sqrt{t}-1)^{2} \xi\right) \mathrm{d} \xi=0
$$


where $t>1$. We have

$$
\limsup _{t \rightarrow \infty} \int_{g(t, \beta)}^{t} \Theta(s) \int_{g(s, \beta)}^{g(t, \beta)} \frac{1}{r_{1}(u)} \int_{u}^{g(t, \beta)} \frac{1}{r_{2}(v)} \mathrm{d} v \mathrm{~d} u \mathrm{~d} s=\frac{4}{3}
$$

and $(1-p) \limsup _{u \rightarrow 0} \frac{u}{f(u)}=\frac{1}{2}<\frac{4}{3}$. Thus, all conditions of Corollary 2.1 are satisfied then all bounded solutions of the above equation are oscillatory.

Remark 2.1 If $\alpha=0, \beta=1, q(t, \xi)=q(t), g(t, \xi)=g(t)$ and $\sigma(\xi)=\xi$ then, our results extend the results in [13].

\subsection{Oscillation Criteria If $\mathrm{z}(t)=x(t)-p x(\tau(t))$}

In this section, we will present some oscillation criteria for Equation (1) under the case $z(t)=x(t)+p(t) x(\tau(t))$ and the condition

$$
\frac{f(u)}{u} \geq k>0 \text { for } u \neq 0 \text { and } t \geq t_{0}
$$

Lemma 2.2 If $x(t)$ is an eventually positive solution of (1), then for sufficiently large t, there are only two possible cases:

(i) $z(t)>0, z^{\prime}(t)<0$ and $\left(r_{1}(t) z^{\prime}(t)\right)^{\prime}>0$

(ii) $z(t)>0, z^{\prime}(t)>0$ and $\left(r_{1}(t) z^{\prime}(t)\right)^{\prime}>0$.

Proof. The proof of this lemma is similar to the proof Lemma 1 in [9] and we omit the details.

Theorem 2.3 Let (16) holds. If

$$
\int_{t_{0}}^{\infty} \frac{1}{r_{1}(v)} \int_{v}^{\infty} \frac{1}{r_{2}(u)} \int_{u}^{\infty} \Theta(s) \mathrm{d} s \mathrm{~d} u \mathrm{~d} v=\infty,
$$

and there exist a positive real function $\rho(t)$ such that

$$
\limsup _{t \rightarrow \infty} \int_{t_{0}}^{t}\left(k \rho(s)(1-p) \Theta(s)-\frac{\rho^{\prime 2}(s) r_{1}(g(s, a))}{4 \rho(s) R(g(s, a)) g^{\prime}(s, a)}\right) \mathrm{d} s=\infty
$$

Then every solution of Equation (1) is either oscillatory or tends to zero.

Proof. Let $x$ be a non-oscillatory solution of Equation (1) on the interval $I$. Without loss of generality we may assume that $x(t)>0$. Then there exists a $t_{1} \geq t_{0}$ such that $x(t)>0, x(\tau(t))>0$ and $x(g(t, \xi))>0$ for $t \geq t_{1}$. By Lemma 2.2, we have two cases for $z(t)$. In the Case (i), since $z(t)>0$ and $z^{\prime}(t)<0$, we get $\lim _{t \rightarrow \infty} z(t)=\mu \geq 0$. Let $\mu>0$, then we have $\mu<z(t)<\mu+\varepsilon$ for all $\varepsilon>0$ and $t$ enough large. Choosing $\varepsilon<\frac{1-p}{p} \mu$, we obtain

$$
\begin{aligned}
x(t) & =z(t)-p(t) x(\tau(t)) \\
& \geq \mu-p z(\tau(t)) \geq L(\mu+\varepsilon) \geq L z(t) .
\end{aligned}
$$

where $L=\frac{\mu-p(\mu+\varepsilon)}{\mu+\varepsilon}>0$. Hence, from (1), ( $\left.\mathrm{A}_{6}\right)$ and (16), we have

$$
\left(r_{2}(t)\left(r_{1}(t) z^{\prime}(t)\right)^{\prime}\right)^{\prime} \leq-k L \int_{\alpha}^{\beta} q(t, \xi) z(g(t, \xi)) \mathrm{d} \sigma(\xi)<-k L \mu \Theta(t) .
$$

By integrating two times from $t$ to $\infty$, we get 


$$
-z^{\prime}(t)>k L \mu \frac{1}{r_{1}(t)} \int_{t}^{\infty} \frac{1}{r_{2}(u)} \int_{u}^{\infty} \Theta(s) \mathrm{d} s \mathrm{~d} u
$$

Integrating the last inequality from $t_{1}$ to $\infty$, we obtain

$$
z\left(t_{1}\right)>k L \mu \int_{t_{1}}^{\infty} \frac{1}{r_{1}(v)} \int_{v}^{\infty} \frac{1}{r_{2}(u)} \int_{u}^{\infty} \Theta(s) \mathrm{d} s \mathrm{~d} u \mathrm{~d} v
$$

This contradicts to the condition (17), then $\lim _{t \rightarrow \infty} z(t)=0$, which implies that $\lim _{t \rightarrow \infty} x(t)=0$. In the Case (ii), since $z(t)>0$ and $z^{\prime}(t)>0$. Then there exist a $t_{1} \geq t_{0}$ such that

$$
x(t) \geq(1-p) z(t)
$$

for $t \geq t_{1}$. Thus, from (1), $\left(\mathrm{A}_{4}\right)$ and $\left(\mathrm{A}_{6}\right)$, we get

$$
\left(r_{2}(t)\left(r_{1}(t) z^{\prime}(t)\right)^{\prime}\right)^{\prime} \leq-k(1-p) z(g(t, a)) \Theta(t) .
$$

Also, we have

$$
r_{1}(t) z^{\prime}(t)=r_{1}\left(t_{1}\right) z^{\prime}\left(t_{1}\right)+\int_{t_{1}}^{t} \frac{r_{2}(s)\left(r_{1}(s) z^{\prime}(s)\right)^{\prime}}{r_{2}(s)} \mathrm{d} s \geq r_{2}(t)\left(r_{1}(t) z^{\prime}(t)\right)^{\prime} \int_{t_{1}}^{t} \frac{1}{r_{2}(s)} \mathrm{d} s,
$$

Since $\left(r_{2}(t)\left(r_{1}(t) z^{\prime}(t)\right)^{\prime}\right)^{\prime} \leq 0$, we obtain

$$
z^{\prime}(g(t, a)) \geq \frac{r_{2}(t)\left(r_{1}(t) z^{\prime}(t)\right)^{\prime}}{r_{1}(g(t, a))} R(g(t, a))
$$

Now, we define

$$
\omega(t)=\rho(t) \frac{r_{2}(t)\left(r_{1}(t) z^{\prime}(t)\right)^{\prime}}{z(g(t, a))}
$$

By differentiating and using (19) and (20), we get

$$
\begin{aligned}
\omega^{\prime}(t) \leq & -k \rho(t)(1-p) \Theta(t)+\frac{\rho^{\prime}(t)}{\rho(t)} \omega(t)-\frac{R(g(t, a)) g^{\prime}(t, a)}{\rho(t) r_{1}(g(t, a))} \omega^{2}(t) \\
= & -k \rho(t)(1-p) \Theta(t)+\frac{\rho^{\prime 2}(t) r_{1}(g(t, a))}{4 \rho(t) R(g(t, a)) g^{\prime}(t, a)} \\
& -\left(\sqrt{\frac{R(g(t, a)) g^{\prime}(t, a)}{\rho(t) r_{1}(g(t, a))}} \omega(t)-\rho^{\prime}(t) \sqrt{\frac{r_{1}(g(t, a))}{4 \rho(t) R(g(t, a)) g^{\prime}(t, a)}}\right)^{2} .
\end{aligned}
$$

Hence, we obtain

$$
\omega^{\prime}(t) \leq-k \rho(t)(1-p) \Theta(t)+\frac{\rho^{\prime 2}(t) r_{1}(g(t, a))}{4 \rho(t) R(g(t, a)) g^{\prime}(t, a)} .
$$

By integrating the above inequality from $t_{1}$ to $t$ we have

$$
\omega(t) \leq \omega\left(t_{1}\right)-\int_{t_{1}}^{t}\left(k \rho(s)(1-p) \Theta(s)-\frac{\rho^{\prime 2}(s) r_{1}(g(s, a))}{4 \rho(s) R(g(s, a)) g^{\prime}(s, a)}\right) \mathrm{d} s .
$$


Taking the superior limit as $t \rightarrow \infty$ and using (18), we get $\omega(t) \rightarrow-\infty$ which contradicts that $\omega(t)>0$. This completes the proof of Theorem 2.3.

Remark 2.2 We can rewrite the condition (17) in the Theorem 2.3 as following

$$
\int_{t_{0}}^{\infty}\left(\int_{t_{0}}^{r} \frac{\mathrm{d} v}{r_{1}(v)}\left(\int_{v}^{r} \frac{\mathrm{d} u}{r_{2}(u)}\right)\right) \Theta(r) \mathrm{d} r=\infty .
$$

Remark 2.3 If $\tau(t)=t-\tau$ and $f(x)=x$, then our results extend the results in [3].

Example 2.2 Consider the differential equation

$$
\left(\frac{1}{t}\left(\frac{1}{t} z^{\prime}(t)\right)^{\prime}\right)^{\prime}+\int_{0}^{1} \frac{t^{2} \mathrm{e}^{t^{2} \xi}}{\mathrm{e}^{t^{2}}-1} x(\xi(t-1))\left(x^{2}(\xi(t-1))+2\right) \mathrm{d} \xi=0,
$$

where $\dot{t}>1$ and $z(t)=x(t)+\frac{1}{2} x(t-1)$. Choosing $\rho(t)=1$ and $k=2$. Thus, all conditions of Theorem 2

are satisfied then every solutions of this equation is either oscillatory or tends to zero.

\section{References}

[1] Agarwal, R.P., Aktas, M.F. and Tiryaki, A. (2009) On Oscillation Criteria for Third Order Nonlinear Delay Differential Equations. Archivum Mathematicum (Brno), 45, 1-18.

[2] Aktas, M.F., Tiryaki, A. and Zafer, A. (2010) Oscillation Criteria for Third-Order Nonlinear Functional Differential Equations. Applied Mathematics Letters, 23, 756-762. http://dx.doi.org/10.1016/j.aml.2010.03.003

[3] Candan, T. and Dahiya, R.S. (2005) Functional Differential Equations of Third Order. Electronic Journal of Differential Equations, Conference 12, 47-56.

[4] Dzurina, J. and Kotorova, R. (2008) Asymptotic Properties of Trinomial Delay Differential Equations. Archivum Mathematicum (Brno), 44, 149-158.

[5] Dzurina, J. and Kotorova, R. (2009) Properties of the Third Order Trinomial Differential Equations with Argument. Nonlinear Analysis, 71, 1995-2002. http://dx.doi.org/10.1016/j.na.2009.01.070

[6] Elabbasy, E.M., Hassan, T.S. and Moaaz, O. (2012) Oscillation Behavior of Second Order Nonlinear Neutral Differential Equations with Deviating Arguments. Opuscula Mathematica, 32, 719-730.

[7] El-sheikh, M.M., Sallam, R. and Mohamady, N. (2013) On the Oscillation of Third Order Neutral Delay Differential Equations. Applied Mathematics \& Information Sciences Letters, 1, 77-80.

[8] Erbe, L.H., Kong, Q. and Zhang, B.G. (1994) Oscillation Theory for Functional Differential Equations. Marcel Dekker, Inc., New York.

[9] Graef, J.R., Savithri, R. and Thandapani, E. (2002) Oscillatory Properties of Third Order Neutral Delay Differential Equations. Proceedings of the Fourth International Conference on Dynamical Systems and Differential Equations, Wilmington, 24-27 May 2002, 342-350.

[10] Hale, J. (1977) Theory of Functional Differential Equations. 2nd Edition, Applied Mathematical Sciences Vol. 3, Springer, New York. http://dx.doi.org/10.1007/978-1-4612-9892-2

[11] Su, M. and Xu, Z. (2012) Oscillation Criteria Of Certain Third Order Neutral Differential Equations. Differential Equations \& Applications, 4, 221-232. http://dx.doi.org/10.7153/dea-04-13

[12] Li, T., Thandapani, E. and Graef, J.R. (2012) Oscillation of Third-Order Neutral Retarded Differential Equations. International Journal of Pure and Applied Mathematics, 75, 511-520.

[13] Mihalikova, B. and Kostikova, E. (2009) Boundedness and Oscillation of Third Order Neutral Differential Equations, Tatra Mountains Mathematical Publications, 43, 137-144. http://dx.doi.org/10.2478/v10127-009-0033-6

[14] Jaros, J. and Kusano, T. (1990) On a Class of First Order Nonlinear Functional Differential Equations of Neutral Type. Czechoslovak Mathematical Journal, 40, 475-490. 